\title{
Thermoluminescence of simulated interstellar matter after gamma-ray irradiation
}

\section{Forsterite, enstatite and carbonates}

\author{
K. Koike ${ }^{1}$, M. Nakagawa ${ }^{1}$, C. Koike ${ }^{2}$, M. Okada ${ }^{3}$, and H. Chihara ${ }^{4,2}$ \\ 1 Faculty of Education, Kagawa University, Takamatsu 760-8522, Japan \\ e-mail: koike@ed.kagawa-u.ac.jp \\ 2 Kyoto Pharmaceutical University, Kyoto 607-8412, Japan \\ 3 Research Reactor Institute, Kyoto University, Kumatori 590-0499, Japan \\ ${ }^{4}$ Department of Earth and Space Science, Osaka University, Toyonaka, Osaka 560-0043, Japan
}

Received 22 June 2001 /Accepted 9 April 2002

\begin{abstract}
Interstellar matter is known to be strongly irradiated by cosmic radiation and several types of cosmic ray particles. Simulated interstellar matter, such as synthesized forsterite $\left(\mathrm{Mg}_{2} \mathrm{SiO}_{4}\right)$, enstatite $\left(\mathrm{MgSiO}_{3}\right)$ and magnesite $\left(\mathrm{MgCO}_{3}\right)$, has been irradiated with ${ }^{60} \mathrm{Co}$ gamma-rays in liquid nitrogen, and also irradiated with fast neutrons at $10 \mathrm{~K}$ and $70 \mathrm{~K}$ by making use of the low-temperature irradiation facility of the Kyoto University Reactor (KUR-LTL. Maximum fast neutron dose is $10^{17} n_{\mathrm{f}} / \mathrm{cm}^{2}$ ). After irradiation, samples are stored in liquid nitrogen for several months to allow the decay of induced radioactivity. We measured the luminescence spectra of the gamma ray irradiated samples during warming to $370 \mathrm{~K}$ using a spectrophotometer. For the forsterite and magnesite, the spectra exhibit a rather intense peak at about $645-655 \mathrm{~nm}$ and $660 \mathrm{~nm}$ respectively, whereas luminescence scarcely appeared in the natural olivine sample. The spectra of forsterite is very similar to the ERE of the Red Rectangle.
\end{abstract}

Key words. ISM: dust, extinction - ISM: general - ISM: lines and bands

\section{Introduction}

Interstellar matter is irradiated by both electromagnetic energy and cosmic ray particles, including gamma rays, neutrons, protons, heavy-ions, etc. Irradiation will cause some changes in these materials, especially to their optical properties. Investigation of this problem is expected to advance our understanding of interstellar and circumstellar matter. In particular, it is well known that extremely large fluxes of neutrons and gamma-rays are emitted during supernova explosions and during the Hayashi-phase in the early stage of protostellar systems. Moreover, interplanetary dust is often irradiated by gamma rays and fast neutrons during periods of flare activity on the sun, which is repeated often. Grains in interstellar and circumstellar space are typically at extremely low temperatures and are always irradiated over cosmological time-scales.

Though little is known about the irradiation environment outside of our solar system, it is natural to suppose that there are regions with sufficient radiation flux to cause thermoluminescence, for example, a region near a supernova explosion or a source of gamma-ray emission, and are exposed for a long time at extremely low temperature. The effect of this radiation will accumulate in the grains within this low temperature

Send offprint requests to: $\mathrm{K}$. Koike, e-mail: koike@ed.kagawa-u.ac.jp environment, and might be observed provided that the conditions to release the accumulated energy can be realized in circumstellar space.

The effects of irradiation on simple single crystals such as $\mathrm{SiO}_{2}, \mathrm{CaCO}_{3}$ and $\mathrm{CaF}_{2}$ have been investigated by several authors (Nakagawa et al. 1988; Nakagawa et al. 1999) from the viewpoint of solid state physics or material science. However, irradiation of materials of interstellar matter has not been studied. It may thus be worthwhile to investigate the effects of irradiation on simulated interstellar matter such as forsterite, enstatite and carbonates. Forsterite and enstatite have been found by many ISO observations in both young and evolved stars and in our own solar system (Waters et al. 1998, 1998; Malfait et al. 1998; Wooden et al. 1999). Carbonates such as dolomite $\left(\mathrm{CaMg}\left(\mathrm{CO}_{3}\right)_{2}\right)$, breunnerite $\left(\mathrm{Mg}(\mathrm{Fe}, \mathrm{Mn})\left(\mathrm{CO}_{3}\right)_{2}\right)$, calcite $\left(\mathrm{CaCO}_{3}\right)$, and $\mathrm{Mg}$, Ca-bearing siderite $\left(\mathrm{FeCO}_{3}\right)$ were found in CI chondrites (Endress et al. 1996). Especially, it should be noted that the broad emission feature responsible for the extended red emission (ERE) appears around the 500-900 nm region in many reflection nebula, and the Red Rectangle nebula shows sharp emission features over a broad band (Witt \& Boroson 1990). In the Red Rectangle nebulae, both PAH-features and crystalline silicates (forsterite and enstatite) were observed (Waters et al. 1998). The effect of irradiation on the optical properties of simulated interstellar 

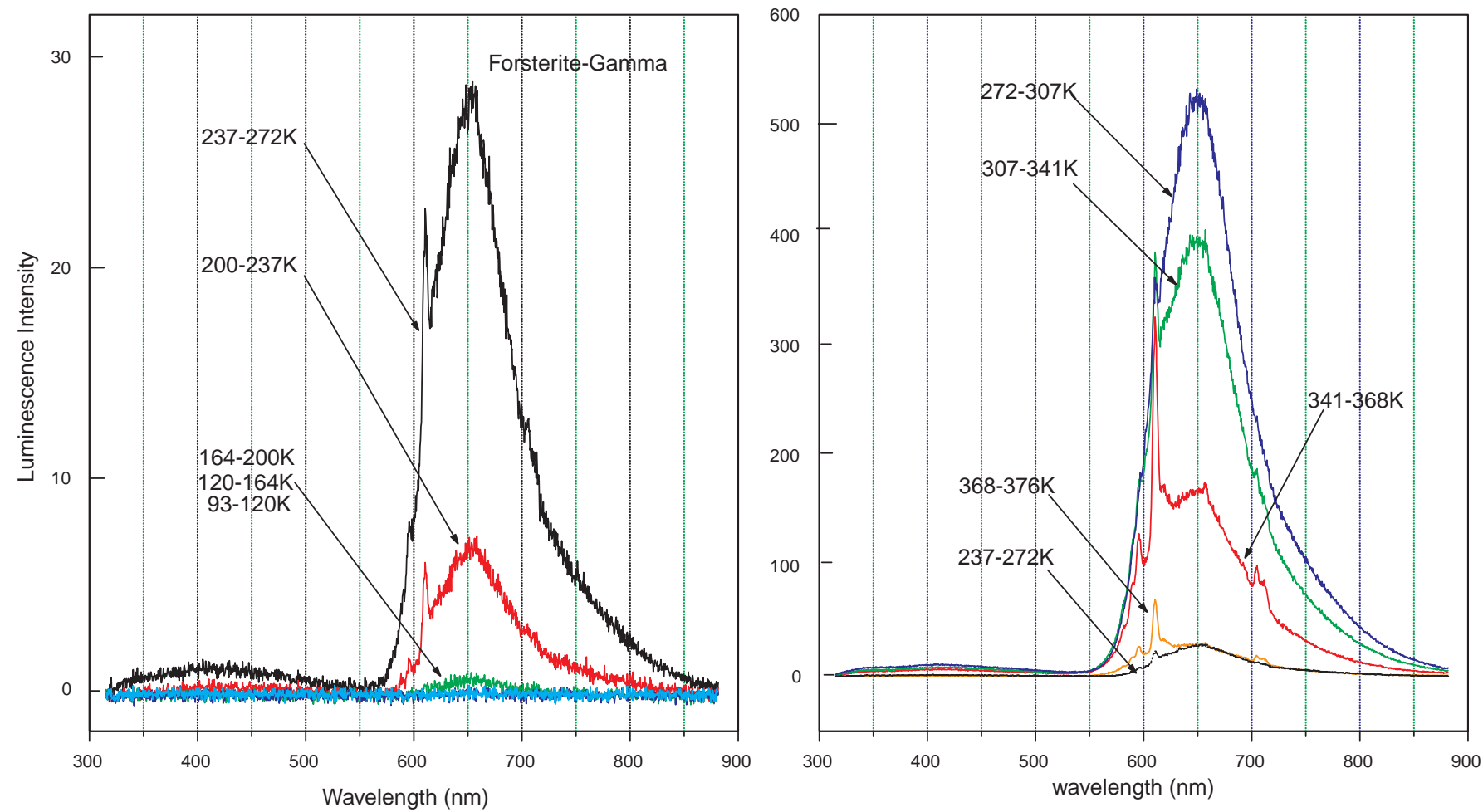

Fig. 1. Thermoluminescence spectra of forsterite $\left(\mathrm{Mg}_{2} \mathrm{SiO}_{4}\right)$ single crystals. The left figure corresponds to $93-272 \mathrm{~K}$ and the right figure 272-376 K.

materials such as forsterite and enstatite by gamma rays and neutrons at low temperature are very interesting.

\section{Features of thermoluminescence spectrum}

Bulk samples such as forsterite, $\mathrm{Mg}_{2} \mathrm{SiO}_{4}$, natural olivine from Egypt, orthoenstatite $\mathrm{MgSiO}_{3}$ and natural magnesite $\mathrm{MgCO}_{3}$ from the Democratic People's Rep. of Korea were irradiated with gamma-rays to a dose of about $10.4 \times 10^{4} \mathrm{~Gy}(\mathrm{~J} / \mathrm{Kg})$ in liquid nitrogen using the ${ }^{60} \mathrm{Co}$ gamma-ray irradiation facility of Kyoto University Reactor. The gamma-rays of ${ }^{60} \mathrm{Co}$ have two peaks at $1.1 \mathrm{MeV}$ and 1.3 MeV. Our samples of forsterite and enstatite were synthesized by Takei \& Kobayashi (1974), and Tachibana (2000) using the CZ (Czochralski) and Flux method, respectively, with high accuracy. The bulk of the irradiated forsterite is rectangular-shaped (about $6 \times 8 \times 15 \mathrm{~mm}$ size, about $1 \mathrm{~mm}$ thick) and its weight is about $138 \mathrm{mg}$. The irradiated enstatite consists of several small fragments (about 1-2 $\mathrm{mm}$ size) and the total weight is about $114 \mathrm{mg}$.

We have measured the thermoluminescence spectra of these samples using a spectrophotometer (including a CCD camera, from Princeton Instruments, Inc.). The sample is put on a thermally-isolated plate, previously cooled to liquid nitrogen temperature. The luminescence emission during warming is introduced to the CCD measuring system using an optically transparent fiber. The head of sensor of the CCD measuring system was settled at the steady point about $3 \mathrm{~cm}$ above each sample. The time spent warming the samples from liquid nitrogen temperature to room temperature $(333 \mathrm{~K})$ was $15 \mathrm{~min}$. It should be noted that this time is sufficient to observe the thermoluminescence, that is, the photons emitted at the thermal equilibrium state. The color of forsterite changed from translucent white to dark-white and -gray (rather dark red-violet) after gamma-ray irradiation.

Figure 1 shows the luminescence spectra of forsterite $\left(\mathrm{Mg}_{2} \mathrm{SiO}_{4}\right)$ after gamma-ray irradiation. It shows a rather intense, broad peak at about $645-655 \mathrm{~nm}$ (a weak, broad peak at $400-440 \mathrm{~nm}$ ), and a sharp peak at $610 \mathrm{~nm}$. The sharp peak at $610 \mathrm{~nm}$ appears as the sample warms from $200 \mathrm{~K}$ to room temperature. Other weak peaks at 590, 595, and $705 \mathrm{~nm}$ appear distinctly above $200 \mathrm{~K}$. The broad peak at $645-655 \mathrm{~nm}$ suddenly increases in intensity and becomes strongest at 272$307 \mathrm{~K}$, while the weak peaks fade into background. Above $307 \mathrm{~K}$, the luminescence gradually becomes weak and other peaks at 595, 605, 705, $710 \mathrm{~nm}$ become prominent. Preliminary data on another sample of irradiated forsterite (its weight about $244 \mathrm{mg}$ ) showed almost the same luminescence spectra, though the earliest luminescence started at a somewhat low temperature. Figure 2 shows the total strength of the luminescence in Fig. 1. A magnification of the emitted thermoluminescence spectrum obtained at the lowest temperature of the range from about 164-200 K in Fig. 1 is shown in Fig. 3.

In contrast, luminescence is scarcely visible in natural olivine (from Egypt, Fo ${ }_{90}$ ). For enstatite, we have detected the luminescence spectra, shown in Fig. 4. Two broad peaks appear at about 545 and $760 \mathrm{~nm}$, and these peaks are very weak compared to the spectra of forsterite. The weight of enstatite used was $114 \mathrm{mg}$, while that of forsterite was $138 \mathrm{mg}$ (discussed above). 
Figure 6 shows the measurement of the luminescence spectra of magnesite, $\mathrm{MgCO}_{3}$, after gamma-ray irradiation. The spectrum of $\mathrm{MgCO}_{3}$ shows a very broad peak at about $660 \mathrm{~nm}$, and this peak becomes strongest at $350-375 \mathrm{~K}$. The irradiated magnesite is a bulk sample (about $5 \times 5 \times 2 \mathrm{~mm}$ in size) and its weight is $93 \mathrm{mg}$. It should be emphasized that the thermoluminescence of $\mathrm{MgCO}_{3}$ is sufficiently strong to be easily visible to the unaided eye. The color of magnesite changed from a translucent white to a rather white-gray with pink-yellow tendency after gamma-ray irradiation.

We have also measured the temperature-dependence of the luminescence of calcite. The luminescence spectrum of calcite is shown in Figs. 8 and 9. The intensity increases as the temperature increases from $77 \mathrm{~K}$; the peak position lies between $620-640 \mathrm{~nm}$ at $300 \mathrm{~K}$. Above $300 \mathrm{~K}$, the intensity increases further, and at $327-337 \mathrm{~K}$ the intensity is strongest, the shape of luminescence spectrum changes and the peak position shifts to around $605 \mathrm{~nm}$. At temperatures higher than $337 \mathrm{~K}$, the intensity becomes weaker.

\section{Luminescent efficiency for each sample}

It should be noted that all data in this paper were measured under almost the same conditions in June 2001, that is, the same device, the same setting and the same warming time of $15 \mathrm{~min}$. The only exception is the warming time of enstatite rather long (that is $18 \mathrm{~min}$ ) in order to detect weaker signals. The reappearance of all data of gamma-ray irradiated samples was confirmed compared with the preliminary data measured in September and October 2000.

The measurement of absolute intensity of luminescence is very difficult, so our measurements express the relative intensity under almost the same conditions. It should be noted that the background fluctuations of luminescence are of about the order of 0.2-0.3 units of intensity in Figs. 3, 4 and 6. We have confirmed that the background fluctuation level of $\mathrm{CaCO}_{3}$ is also $0.2-0.3$ units in Fig. 8. The comparison of relative intensity of luminescence with the background fluctuations will give a clue about the absolute quantity of intensity.

Measurement of the absolute intensity of luminescence is further problem.

\section{Related problems and the other measurements}

We also investigated the effect of irradiation both on a bulk sample and on particle samples embedded in polyethylene using neutrons at a dose of $8 \times 10^{16} n_{\mathrm{f}} / \mathrm{cm}^{2}$ at both $10 \mathrm{~K}$ and $70 \mathrm{~K}$ using the low-temperature irradiation facility of Kyoto University Reactor (KUR-LTL: Low Temperature Loop) (Okada et al. 2001). After neutron irradiation for 75 hours, samples are stored in liquid nitrogen for several months to await the decay of induced radioactivity.

We measured the absorption coefficient of neutronirradiated forsterite and enstatite particles using a FTIR spectrometer (Nicolet, Nexus 670) over the wavelength range from $30-100 \mu \mathrm{m}$. The samples were irradiated at $70 \mathrm{~K}$ using neutrons in September, 2000. The gross features of the spectrum show no apparent differences from the spectrum

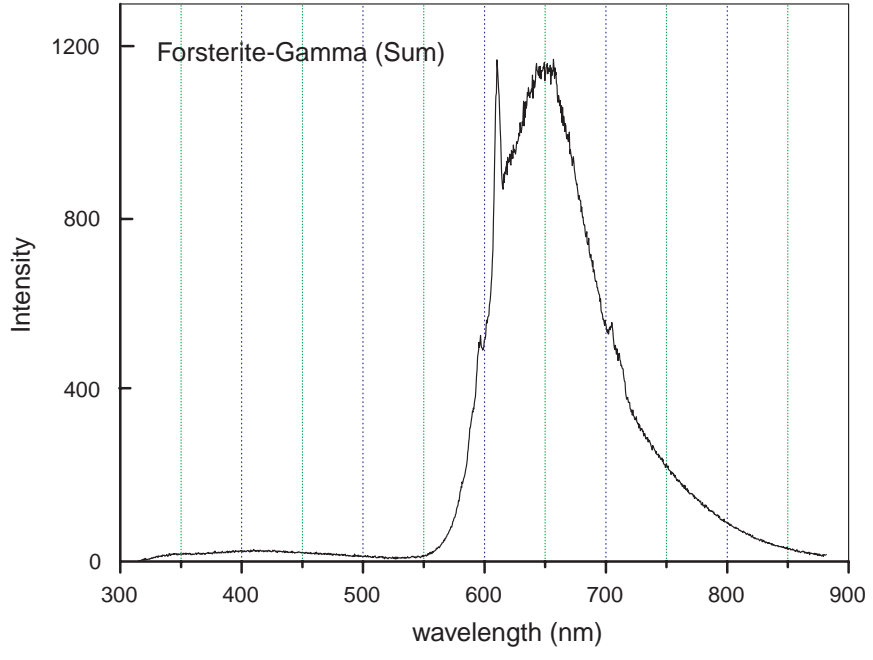

Fig. 2. The sum of total thermoluminescence spectra of forsterite in Fig. 1 The peak of the thermoluminescence of $\mathrm{Mg}_{2} \mathrm{SiO}_{4}$ is about $645-655 \mathrm{~nm}$.

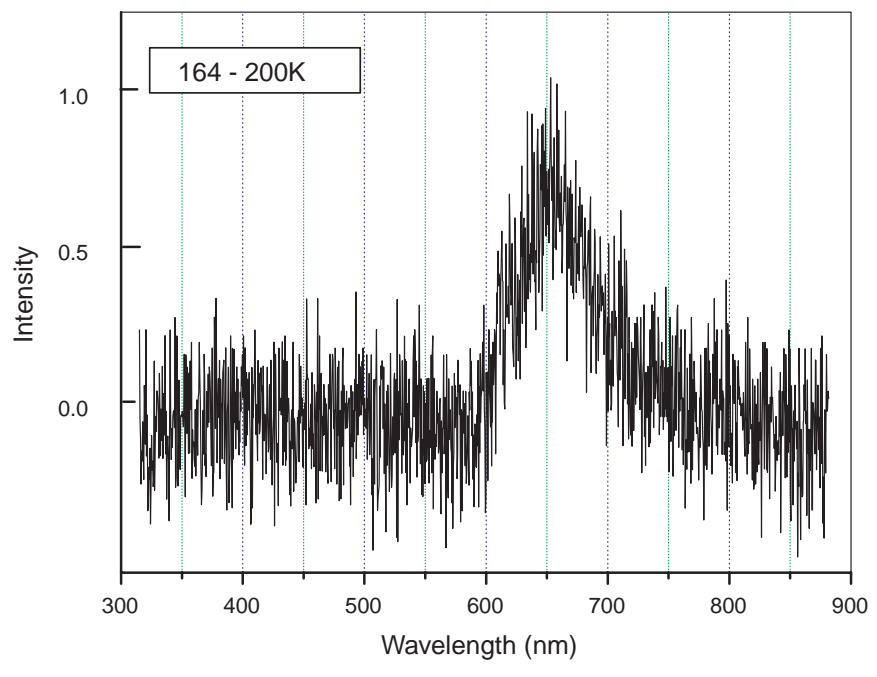

Fig. 3. The earliest (low temperature) thermoluminescence spectra of forsterite at $164-200 \mathrm{~K}$. The peak is at about $645-655 \mathrm{~nm}$.

(Koike et al. 2000, 2000) taken before irradiation. One of the reasons for this result may be that the fast neutrons strongly collide with hydrogen atoms in the polyethylene and only weakly collide with sample particles dispersed in polyethylene.

We have also measured the thermoluminescence spectrum of a neutron-irradiated bulk sample of forsterite, which is shown in Fig. 5. At low temperature, two peaks appeared at about 450 and $650 \mathrm{~nm}$, but above $253 \mathrm{~K}$ the peak at $450 \mathrm{~nm}$ became weak. The peak at $650 \mathrm{~nm}$ appears to be almost the same shape as the peak produced in the gamma-ray irradiated case, however, the strength of the luminescence is considerably weaker. The reason for this difference is not yet known.

For further irradiation experiments using a stronger beam of neutrons in the center of the reactor, we are interested in an accurate measurement of the impurity component in our forsterite sample, because impurities with long half lives cause serious difficulties in measurements of irradiated samples. Previously, Takei \& Kobayashi (1974) reported that spectrographic analysis shows that the forsterite sample is pure. 


\section{Enstatite-Gamma}
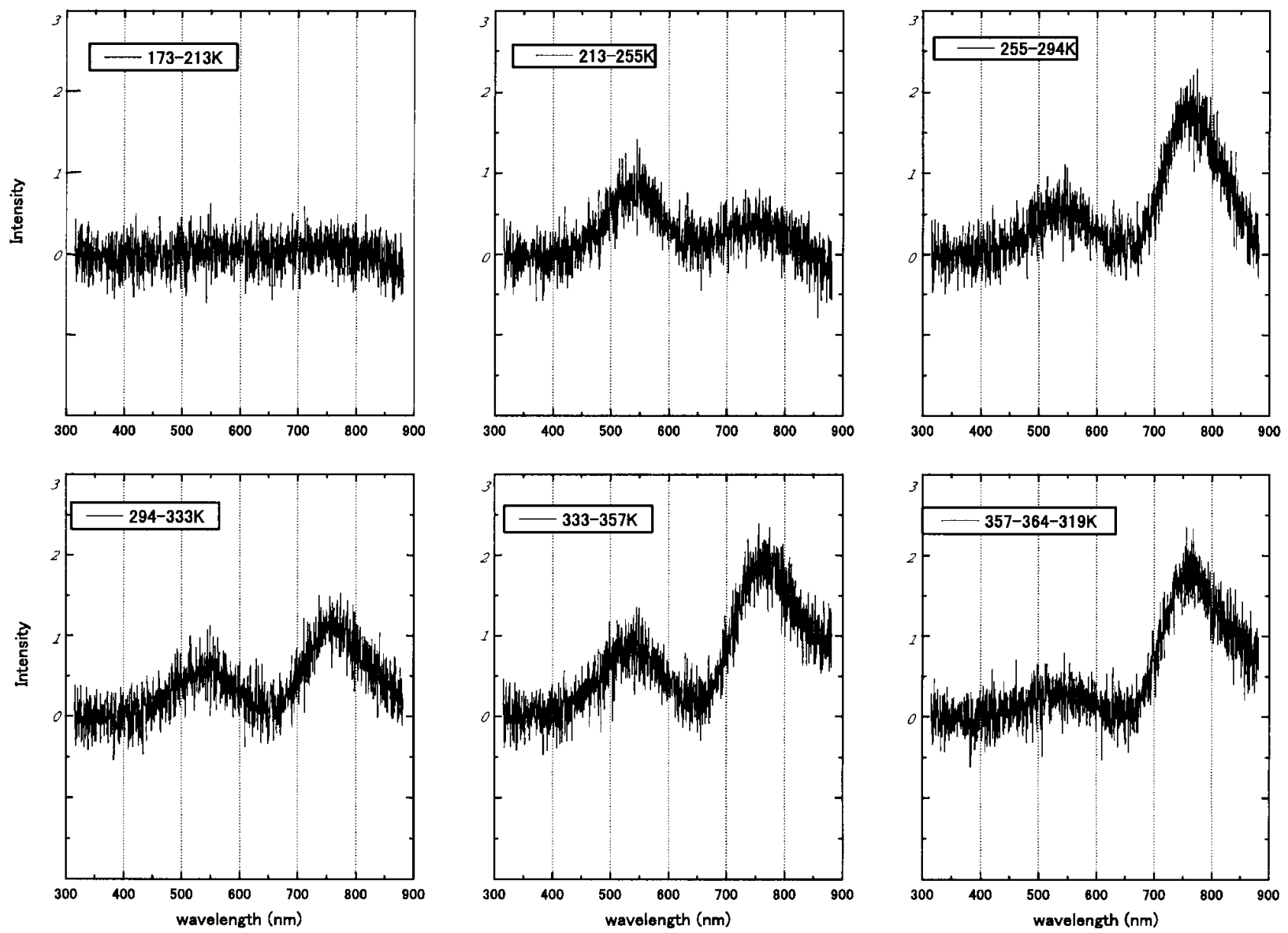

Fig. 4. Thermoluminescence spectra of enstatite $\left(\mathrm{MgSiO}_{3}\right)$ single crystals. (The right figure shows the luminescence when the temperature rose from $357 \mathrm{~K}$ to maximum $364 \mathrm{~K}$, and $319 \mathrm{~K})$.

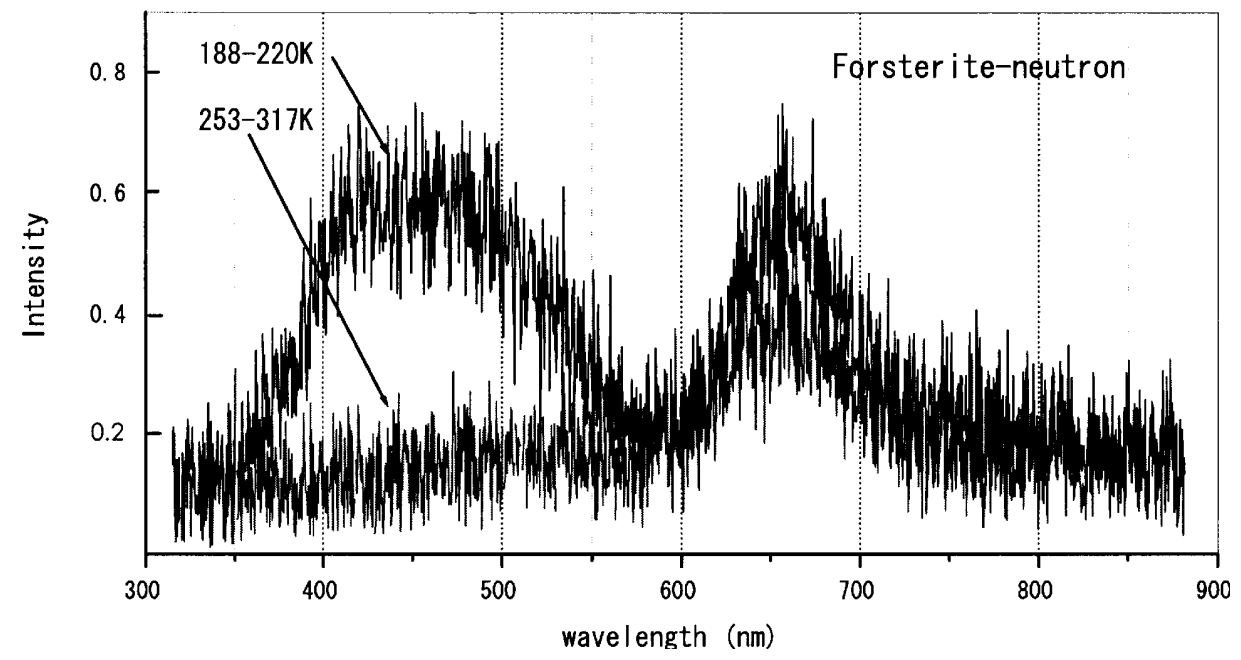

Fig. 5. The thermoluminescence spectra of forsterite after neutron irradiation at $10 \mathrm{~K}$.
However, neutron activation analysis reveals that it contains an infinitesimal quantity of Ir at about 16-18 wt ppm. This level of Ir impurity is below the detection limit of spectrographic analysis. We have also measured our sample using radio activation analysis, and confirmed that our sample is almost pure; that is, other elements except for $\mathrm{Mg}, \mathrm{Si}, \mathrm{O}$ and an infinitesimal quantity of Ir are not detected.

\section{Similarity to ERE of the Red Rectangle spectrum}

It is a remarkable fact that the observed spectrum of ERE (Extended Red Emission) of the Red Rectangle (Witt \& Boroson 1990) given in Fig. 7 shows several characteristic features. For carriers of ERE, a possible size effect in crystalline silicon nanoparticles has been discussed (Ledoux et al. 2001). 


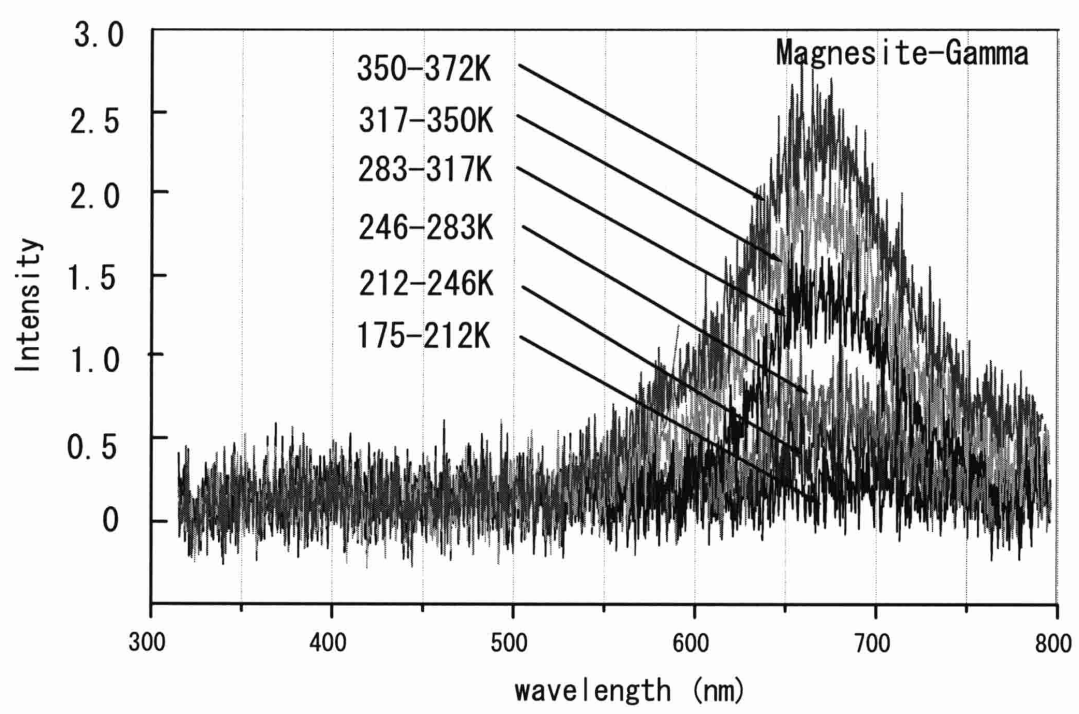

Fig. 6. Thermoluminescence spectra of magnesite $\left(\mathrm{MgCO}_{3}\right)$. The peak is at about $660 \mathrm{~nm}$.

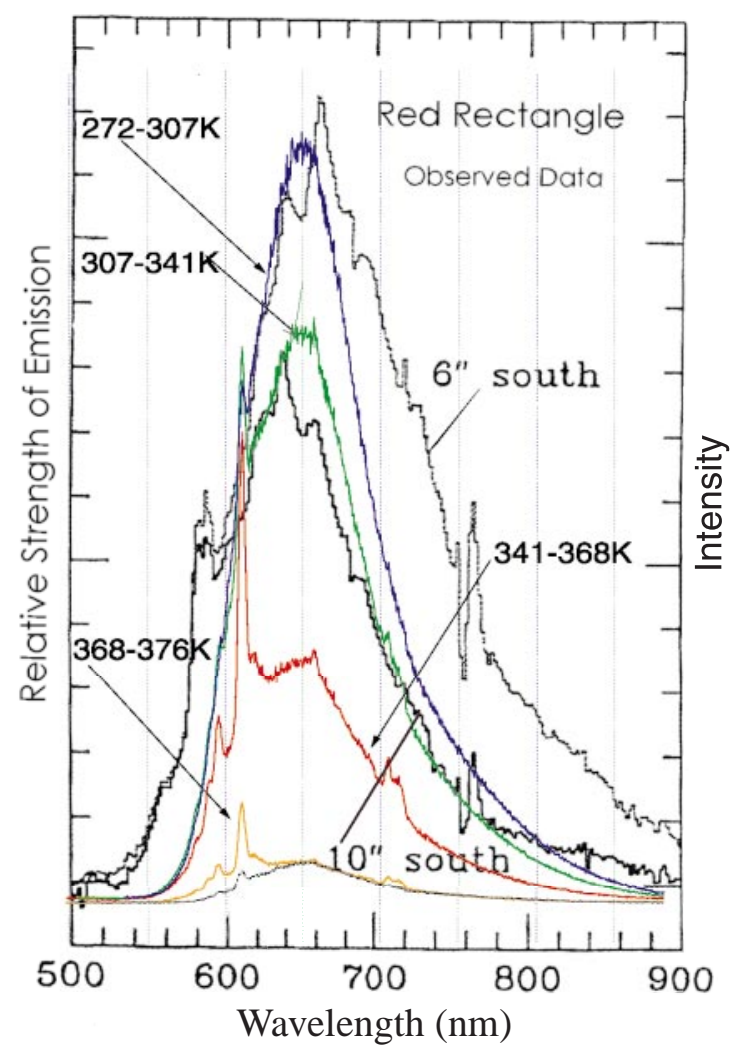

Fig. 7. Comparison with Observation Data of Red Rectangle Spectrum (From Witt \& Boroson 1990) and Forsterite Spectrum (the right-side of Fig. 1).

It has been shown that the photoluminescence spectra of these nanoparticles can explain the gross structure of the ERE spectrum, such as the peak position and the full width at half maximum, etc. However, evidence for the existence of crystalline silicon has not yet been observed. (Li \& Draine 2001, 2002)

It is interesting that the luminescence of forsterite is very similar to ERE of the Red Rectangle (peak at 652-684 nm,



Fig. 8. Typical thermoluminescence spectrum of calcite $\left(\mathrm{CaCO}_{3}\right)$ at $227-300 \mathrm{~K}$. The peak of $\mathrm{CaCO}_{3}$ is at about $640 \mathrm{~nm}$.

half width 70-90 nm). Generally, the ERE from interstellar dust consists of a broad, featureless emission band peaking anywhere from $610-820 \mathrm{~nm}$, with a width of 60-100 nm. The Red Rectangle shows strong emission at least one order of magnitude stronger and exhibits the characteristic features of forsterite. Furthermore, crystalline silicates, such as forsterite, and PAH dust had been observed in the Red Rectangle by ISO observations. The present irradiated forsterite also shows very strong luminescence (peak at 640$660 \mathrm{~nm}$ ) at about 280-308 K with a half-width of about $100 \mathrm{~nm}$, and additional characteristic peaks at 590, 595, and $705 \mathrm{~nm}$.

It should also be remembered that forsterite and enstatite have been found by many ISO observations in many oxygen-rich young and evolved stars. However, ERE is also observed in many carbon-rich stars. It is important to note that the Red Rectangle is a very interesting object with both carbon- and oxygen-rich characteristics, that is, both forsterite and PAH are observed. 


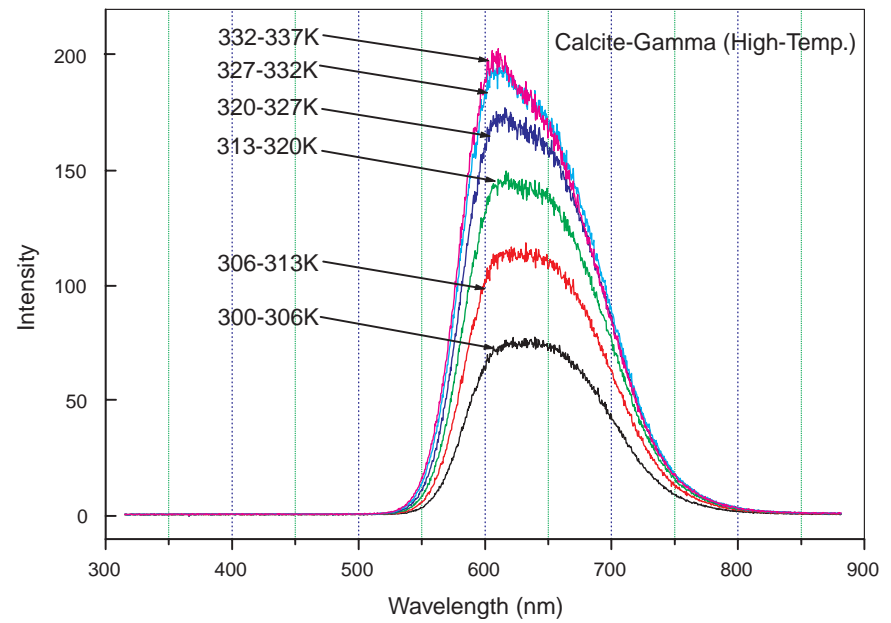

Fig. 9. Typical thermoluminescence spectrum of calcite $\left(\mathrm{CaCO}_{3}\right)$ at $300-337 \mathrm{~K}$. The peak shifts towards $600 \mathrm{~nm}$.

It should be emphasized that our thermoluminescence spectrum of forsterite peaks at about 645-655 $\mathrm{nm}$ and at $590 \mathrm{~nm}$ is very similar to the ERE of the Red Rectangle.

\section{Discussion}

Little is known about the irradiation environment, around ERE objects. However, it should be noted that interstellar and circumstellar space is typically at extremely low temperature and is always irradiated by both electromagnetic radiation and by cosmic ray particles over cosmological time-scales. Furthermore, it is well known that extremely large fluxes of neutrons and gamma-rays are emitted during supernova explosions. The effect of this radiation will accumulate in the low temperature environment. It will only be observed provided that the condition to release the accumulated energy is realized in circumstellar space. This may occur when irradiated dust moves to a warmer domain in an interstellar or circumstellar environment.

The accumulated energy in irradiated matter is released by thermoluminescence when the matter is warmed. The rate of this release is dominated by the Boltzmann factor, and at extremely low temperatures this rate is practically infinite. In connection with this problem, it is interesting that the thermoluminescence spectrum of particles from the Tyrrhenian Sea exhibits typical spectrum peaks at the layers deposited in the years 1006, 1054, 1181 etc., corresponding to the years of supernova explosions (Castagnoli et al. 1982). In their estimate, the energy emitted during the explosions of the Crab nebula in the year 1054 was about $10^{47} \mathrm{~J}$ and the estimated flux of energy at a point of distance of the radius of the Galaxy is about $10^{5} \mathrm{~J} / \mathrm{m}^{2}$.

Though most of the energy of such explosions are carried by neutrinos, a certain fraction will be carried by X-and gamma rays. It is possible that in extremely low-temperature environments such as interstellar or circumstellar space, the effects of irradiation are "frozen" and could be stable for extremely long times. Therefore various irradiation effects, such as often observed supernova explosions and cosmic rays, might be accumulated in the solid.

The irradiation of matter generally causes lattice defects in crystals, and a certain kind of this effect is observed in thermoluminescence. It is known in many experiments that irradiation effects in solids can be erased by annealing it to several hundred degrees. In other words, the effects of irradiation remain locked into the crystal below such temperatures and may be observed still in effects other than thermoluminescence.

\section{Summary}

In this paper, we report the thermoluminescence spectra for gamma-ray irradiation of some simulated interstellar materials. Forsterite $\left(\mathrm{Mg}_{2} \mathrm{SiO}_{4}\right)$, enstatite $\left(\mathrm{MgSiO}_{3}\right)$ and magnesite $\left(\mathrm{MgCO}_{3}\right)$ exhibit interesting spectra that bear some resemblance to ERE observed in several astrophysical environments.

We have emphasized that the thermoluminescence of forsterite appears at low temperature (about 160-200 K). In particular, it should be emphasized that the spectrum of forsterite peaks at about $645-655 \mathrm{~nm}$ and at $590 \mathrm{~nm}$ is very similar to the ERE of the Red Rectangle. (Witt \& Boroson 1990) It should be noted that possible size effects of crystalline silicon nanoparticles have been proposed to explain the gross structure of ERE spectra (Ledoux et al. 2001). However, this model can explain only the gross structure of the spectra of ERE sources, and seems unable to explain the characteristic features observed at $590 \mathrm{~nm}$. The most interesting feature of the size effect is that the peak of luminescence shifts depending on the size of the nanoparticles. It may be an interesting problem to examine the possible existence of similar size effects in forsterite nanoparticles.

Finally, the irradiation of matter is expected to cause various effects on solidmatter in addition to thermoluminescence. Investigating these effects in the context of astrophysics is an additional problem.

Acknowledgements. The authors would like to express their sincere thanks to Prof. Tsuchiyama, who provided superior samples of simulated interstellar matter such as forsterite and enstatite. We would like also to acknowledge members of Prof. Atobe's group of Naruto University of Education for allowing us to use their equipment including the sample holder with thermo controller.

This work was supported by the KUR projects (12062, 13p1-6). Part of this work was supported by Grant-in-Aid of Japanese Ministry of Education, Science, and Culture (12440054).

\section{References}

Castagnoli, G. C., Bonino, G., \& Miono, S. 1982, Il Nuovo Cimento, $5 \mathrm{C}, 488$

Endress, M., Zinner, E., \& Bischoff, A. 1996, Nature, 379, 701

Koike, C., Tsuchiyama, A., Shibai, H., et al. 2000, A\&A, 363, 1115

Koike, C., Chihara, H., Tsuchiyama, A., et al. 2000, Proc. 33rd ISAS

Lunar and Planet. Symp., 33, 95

Ledoux, G., Guillois, O., Huisken, F., et al. 2001, A\&A, 377, 707

Li, A., \& Draine, B. T. 2001, ApJ, 550, L213 
Li, A., \& Draine, B. T. 2002, ApJ, 564, 803

Malfait, K., Waelkens, C., Waters, L. B. F. M., et al. 1998, A\&A, 332, L25

Nakagawa, M., Fukunaga, M., Okada, M., et al. 1988, J. Lum., $40 \& 41,345$

Nakagawa, M., Koike, K., Okada, M., et al. 1999, KURRI Prog. Rep., 77

Okada, M., Kanazawa, S., Nozaki, T., et al. 2001, Nuclear Instruments and Methods in Physics Research A, 463, 213
Tachibana, S. 2000, Ph.D. Thesis, Osaka University

Takei, H., \& Kobayashi, T. 1974, J. Cryst. Growth, 23, 121

Waters, L. B. F. M., Beintem, D. A., Zijlstra, A. A., et al. 1998, A\&A, 331, L61

Waters, L. B. F. M., Waelkens, C., Van Winckel, H., et al. 1998, Nature, 391, 868

Witt, A. N., \& Boroson, T. A. 1990, ApJ, 355, 182

Wooden, D. H., Harker, D. E., Woodward, C. E., et al. 1999, ApJ, 517, 1034 